\title{
Les dérives des marchés financiers
}

\author{
Martin Gauthier ${ }^{a}$, Vincent Morin ${ }^{\text {b }}$
}

RÉSUMÉ. Depuis les quarante dernières années, les marchés financiers se sont développés à un rythme fulgurant. Plusieurs facteurs peuvent expliquer cette croissance. Certes, la baisse des taux d'intérêt sur les placements traditionnels aura incité les épargnants à aller vers des placements offrant un plus grand potentiel de rendement. La mondialisation aura aussi permis l'accès à davantage de produits et de marchés. Il ne faut toutefois pas négliger le rôle de la déréglementation du secteur financier qui aura permis aux institutions financières d'offrir une panoplie de services et de créer de nouveaux produits de plus en plus complexes. Tous ces facteurs, combinés à l'émergence de nombreux fonds spéculatifs, auront permis de créer une économie qui repose davantage sur l'industrie financière que sur l'industrie manufacturière.

ABSTRACT. In the last forty years, financial markets have grown at a very quick. pace. A lot of factors can explain this growth. First the low interest rate have encouraged savers to take out their money and place it in more profitable investment. Globalization also gave investors access to new products and markets. But we must not neglect the impact of a low-rules environment wbich has enabled financial institutions to offer a range of services and create new and more complex products. All of these factors combined with the emergence of many bedge funds will have created an economy that relies more on the financial industry than on the manufacturing industry.

\section{Introduction}

La crise financière de 2007 aura fortement ébranlé les marchés financiers et démontré de façon éloquente les failles du système actuel. Certes, le crédit " subprime » est clairement identifié comme le grand responsable de la crise, mais il n'est que le symptôme de problèmes encore plus grands. Si on veut éviter que l'histoire ne se répète, il faut s'attarder aux causes réelles de la crise et proposer des pistes de solutions à long terme. Le but de cet article est de démontrer ce qui a facilité la création des prêts subprime, c'est-à-dire la déréglementation des services financiers, la titrisation des prêts hypothécaires et l'apparition massive de produits dérivés. Nous terminerons en apportant une réflexion sur la place des marchés financiers dans l'économie et sur la nécessité de légiférer davantage pour s'assurer d'éviter une répétition des dérives passées.

\section{Le rôle des marchés financiers}

Les marchés financiers jouent un rôle majeur dans l'économie en facilitant l'accès aux capitaux pour les entreprises, les gouvernements et, indirectement, les particuliers; ce qui permet de favoriser l'investissement et créer davantage de richesse collective. Ces mêmes marchés connaissent des «crises » qui peuvent faire tarir l'accès aux ressources financières. On constate qu'avec l'émergence des produits dérivés, les marchés sont devenus de plus en plus complexes et de plus en plus imprévisibles. L'arrivée massive d'investisseurs privés spéculateurs comme les " hedges funds ", qui visent principalement les profits à court terme, sont venus amplifier les situations de crises. Les banquiers et autres intermédiaires financiers rivalisent d'audace pour créer des produits de plus en plus sophistiqués et parfois incompréhensibles. Dans ce contexte, on peut se demander si les marchés financiers remplissent bien leur fonction et contribuent à la création de richesse collective ou s'ils ne sont tout simplement pas devenus un lieu où quelques investisseurs privilégiés s'enrichissent sur le dos des autres agents économiques.

\footnotetext{
${ }^{a}$ Ancien recteur (2012-2017), professeur, Université du Québec à Chicoutimi

b Directeur DSEA, professeur, Université du Québec à Chicoutimi 


\section{Le marché boursier et le marché hors cote}

Les marchés boursiers ont été créés pour établir un juste prix sur les denrées, les métaux, les actions et plusieurs autres biens tangibles ou titres afin de pouvoir les échanger. Ces marchés boursiers réglementés permettent de s'échanger des titres standardisés afin de réduire l'asymétrie d'information. De fait, ils sont relativement efficaces et il n'est pas facile, même pour l'investisseur chevronné, de s'enrichir plus que la normale.

D'autre part, divers titres financiers ne sont pas échangés sur les marchés boursiers et se négocient plutôt sur des marchés hors cote (OTC Over the Counter). C'est principalement le cas des titres à revenu fixe et de certains produits dérivés. Les marchés OTC sont nettement moins transparents et la plupart des informations ne sont pas disponibles aux particuliers. Ces marchés demeurent imparfaits.

À titre d'exemple, si vous voulez vendre vos actions de la Banque Royale, vous pouvez, à n'importe quel moment, aller sur un site boursier et voir le prix actuel auquel le marché est prêt à acheter vos titres. Si vous placez votre ordre de vente à ce moment, vous savez donc exactement quel prix vous obtiendrez.

Dans le cas des titres à revenu fixe, le prix acheteur et le prix vendeur en cours ne sont pas accessibles au grand public. Dans plusieurs situations, surtout pour les titres moins liquides, c'est le courtier luimême qui vend ses propres titres et qui détermine les prix. Toutefois, le règlement 31-103 sur les obligations et dispenses d'inscription et les obligations continues des personnes inscrites R.R.Q., V-1.1, adopté en 2013 par l'Autorité des marchés financiers du Québec est venu apporter un peu plus de transparence dans ce marché. Le courtier doit désormais divulguer les frais de courtage à son client (Autorité des marchés financiers, 2013).

C’est ce marché, où opèrent une majorité d'investisseurs institutionnels, qui est à l'origine de la plus grande crise financière depuis le krach de 1929. Certes, le surendettement des ménages américains et l'agressivité des banques dans l'octroi du crédit immobilier sont aussi responsables de cette crise financière qui débuta en 2007. Cependant, les prêts à haut risque " subprimes » n'auraient pu exister sans un contexte propice mis en place bien des années auparavant.

\section{Déréglementation du secteur financier}

Dans la foulée de la crise de 1929, le gouvernement américain a instauré une série de réformes pour éviter qu'elle ne se répète dans le futur. La réforme la plus spectaculaire d'entre toutes fut certainement le "Glass-Steagall Act » adopté en 1933 (Chase National Bank of the City of New York, 1933). Cette loi obligeait les banques à faire le choix entre devenir une banque de dépôt ou d'investissement. En somme, si elle choisissait d'être une banque de dépôt, elle pouvait recevoir des dépôts et faire des prêts, mais ne pouvait plus investir dans les valeurs mobilières et vice versa.

Ce cloisonnement a rétabli une certaine confiance dans le système bancaire et empêché les institutions de dépôt d'investir l'argent des déposants dans les marchés boursiers. On a aussi réduit la possibilité de conflits d'intérêts en interdisant à ceux qui accordent des prêts de devenir des conseillers en placement ou à ceux qui vendent des titres boursiers d'accorder du crédit à ces mêmes entreprises. Bref, d'entrer dans des situations d'aléa moral.

À partir des années 1980, une vague de déréglementation a pour résultat de donner davantage de marge de manœuvre aux banques. En fait, l'arrivée successive de gouvernements de droite, notamment aux États-Unis (Ronald Reagan), en GrandeBretagne (Margaret Thatcher) et au Canada (Brian Mulroney) a créé un courant favorable à la libéralisation des marchés financiers (Truffaut, 2017). Ce courant de libéralisation a déclenché une véritable course à la déréglementation. Comme le dit Christopher Johnson, « La City de Londres a souhaité que des réformes soient engagées par souci de gagner la première place dans la ruée internationale à la déréglementation afin de conserver sa réputation de première place financière mondiale » (Johnson, 1993, 227).

Les nombreuses interventions des gouvernements, pour libéraliser les marchés et les rendre plus attrayants pour les institutions financières internationales, auront permis la création de plusieurs nouveaux produits complexes et risqués, notamment les " junk bonds »; des obligations à haut risque qui seront largement utilisées pour financer des offres publiques d'achat. On retiendra aussi des années 1980, la déréglementation qui a touché 
directement les caisses d'épargne en leur permettant d'accroître à un rythme effréné leur nombre de prêts avec comme conséquence qu'à la fin de la décennie, ce sont plusieurs centaines de caisses d'épargne qui seront dissoutes; ce qui amènera le gouvernement américain à éponger une perte de près de 160 milliards de dollars (Truffaut, 2017).

Bref, les années 1980 auront été le théâtre d'un scénario que l'on verra se répéter 20 ans plus tard. Des institutions financières qui décident d'accroitre leur nombre de prêts de façon spectaculaire et des courtiers qui font des centaines de millions de dollars de profit en vendant aux investisseurs des instruments financiers à risque élevé. Finalement, ces actions délétères provoqueront un krach historique où la valeur de l'indice Dow Jones chutera de $23 \%$ en une seule journée. Déjà à cette époque, on avait un aperçu des dangers que pouvait représenter une déréglementation trop grande du secteur financier. Malheureusement, ce ne fut pas suffisant pour arrêter un train bien en marche.

Nous observons de façon particulière que le Canada n'a pas seulement suivi la vague, il a été un précurseur en matière de déréglementation. En effet, en 1987 le gouvernement canadien adoptait le projet de loi C-56 qui proposait un décloisonnement du secteur financier et permettait, entre autres, aux banques de se lancer dans les activités de courtage. En quelques mois seulement plusieurs des plus grandes maisons de courtage du pays sont passées sous le contrôle des banques.

Ce mouvement a donné un avantage aux grandes banques canadiennes qui ont pu concurrencer efficacement leurs rivales américaines sur leur propre marché. Dès le début des années 1990, les banques canadiennes ont commencé à faire des acquisitions répétées sur le marché américain, acquisitions qui ont soulevé l'ire du secteur financier américain. La stratégie agressive du secteur bancaire canadien a mis une énorme pression sur le gouvernement américain pour donner davantage de souplesse aux institutions financières américaines. Ces dernières ont dû attendre 12 ans, soit en 1999, pour qu'une loi similaire soit adoptée aux États-Unis et que l'on enterre définitivement le Glass-Steagall Act. (Chase National Bank of the City of New York, 1933).

La non-réglementation ou la déréglementation des marchés financiers dans plusieurs pays a permis plusieurs dérives. D'ailleurs, chaque citoyen en a payé le prix puisque ces dérapages ont contraint plusieurs gouvernements à multiplier les mesures de soutien pour venir en aide à ces intermédiaires financiers dont la faillite aurait été catastrophique pour l'économie. Ces dérives ont eu un impact majeur, à tel point qu'elles ont menacé la stabilité financière de certains pays et obligé d'autres pays à intervenir pour sauvegarder l'économie mondiale.

Inévitablement, le manque de surveillance et la législation souple ont créé un environnement favorable au développement des institutions financières mondiales et pour comprendre ce qui a pu les pousser à accroître leurs risques de façon aussi importante dans les années 2000, il faut examiner la croissance de la titrisation.

\section{Titrisation des prêts « subprimes »}

Apparue vers la fin des années 1970, la titrisation est un mécanisme qui permet de prendre, par exemple, les prêts qu'accordent les institutions financières et de les regrouper pour former une obligation qui peut être vendue au marché. Le premier titre ainsi créé fut le titre adossé à des hypothèques (Mortgage-backed securities ou MBS). Pour l'investisseur, ce titre était attrayant dans la mesure où l'actif était garanti par une série d'hypothèques de bonne qualité et le rendement offert était supérieur à celui des obligations émises par un État.

Pour l'institution financière, l'avantage était de profiter de l'accès au marché des capitaux afin de lever des fonds pour financer leurs hypothèques plutôt que de se fier uniquement aux déposants traditionnels. L'institution financière pouvait ainsi prêter plus d'argent, donc engranger plus de profits, tout en transférant le risque associé aux prêts vers les détenteurs d'obligations.

Dans les années qui ont suivi, une multitude de produits adossés ont été ainsi créés en regroupant toutes sortes de créances comme celles des cartes de crédit, des prêts-autos, des prêts à la consommation, des créances commerciales et autres. Malgré l'importance de ce marché, les gouvernements ne sont pratiquement pas intervenus pour réglementer ce secteur d'activité puisque ces titres s'échangeaient entre initiés sur des marchés hors-cote. Au contraire, les règlements souples et la volonté du gouvernement américain d'accroître l'accès à la propriété auront été une occasion formidable pour 
les banques de créer deux autres produits, les ABS (Asset Backed Securities) et les CDO (Collateralized Debt Obligation). Ces derniers seront adossés à des hypothèques à haut risque (subprime) et seront en grande partie responsables de la crise qui surgit en 2007.

Avant de parler de ces deux produits, il faut aborder, rapidement, la question du crédit « subprime ». Celuici comprend la tranche des emprunteurs les plus à risque. Ils ont généralement des ratios d'emprunts suffisants, mais peuvent présenter un historique de retard de paiement qui fait diminuer leur cote de crédit. Ce genre de crédit présente un risque de défaut, mais dans la mesure où les prix immobiliers sont constamment à la hausse, la probabilité de perte pour le prêteur peut être relativement faible.

Cependant, au début des années 2000, plusieurs institutions financières américaines vont élargir la définition de « subprime ». Grâce à des taux d'intérêt très faibles, elles ont pu prêter des sommes importantes à des particuliers dont les revenus n'étaient pas très élevés. Elles ont aussi offert des taux d'intérêt promotionnels pour les deux premières années du prêt, permettant ainsi de qualifier des gens pour des prêts dont on savait qu'ils ne seraient jamais capables d'acquitter le versement en temps normal. Pendant cette même période, le nombre de prêts « low documents », des prêts faits sans avoir toutes les preuves de revenus et d'actifs, a tout simplement explosé. Dans le jargon financier, on s'est mis à parler de ces emprunteurs comme des NINJA, pour "No Income, No Job, No Asset ». En résumé, on a prêté des sommes importantes à des individus qui n'avaient pas d'emploi stable, des revenus insuffisants et peu d'historique de crédit.

Plusieurs raisons peuvent expliquer cette importante prise de risque. On croyait à tort que les prix de l'immobilier continueraient de monter et qu'en cas de défaut de paiement, on pourrait récupérer l'argent prêté grâce à la valeur de la maison. On croyait aussi que si l'on possédait des prêts issus de régions différentes, le risque de défaut deviendrait très faible et qu'on pourrait considérablement réduire le risque en misant sur la diversification.

Toutefois, il ne faut pas oublier la véritable raison : c'était très lucratif de le faire. Pour les prêteurs hypothécaires, l'augmentation du volume de prêts leur permettait d'empocher plus de profits. Idem pour les banquiers de Wall Street qui reprenaient ces prêts et les vendaient en paquet sur les marchés financiers, en touchant des centaines de millions de dollars en commissions. C'était également vrai pour les agences de cotation qui attribuaient de généreuses cotes de crédit à ces instruments financiers et obtenaient en retour de juteux honoraires. Ainsi, plus il y avait d'hypothèques, plus il y avait de titres à vendre et comme il y avait d'innombrables acheteurs pour ces titres, il fallait qu'il y ait autant d'hypothèques. Normalement, c'est la demande pour les prêts hypothécaires qui aurait dû amener la création de plus de titres, alors que dans ce cas, c'est plutôt la demande de titres qui a permis la création de plus de prêts et d'en affecter l'offre.

Le premier outil utilisé pour titriser ces prêts à haut risque a été l'ABS «l'Asset-Backed Security ». L'ABS est un titre financier qui génère des intérêts en fonction des versements perçus sur plusieurs prêts de qualité variable. L'ABS peut par la suite être revendu sur le marché à différents investisseurs.

La structure d'un ABS comporte généralement trois parties. La tranche supérieure représente la plus grosse part (entre $60 \%$ à $80 \%$ ). Elle est généralement à très faible risque et est payée en premier. C'est pourquoi elle obtient généralement une cote AAA (la meilleure cote de crédit possible) et rapporte un taux d'intérêt légèrement supérieur à celui des obligations gouvernementales. Ce genre de titre était donc assez facile à vendre sur les marchés, car la demande des investisseurs institutionnels était très forte. La tranche médiane de l'ABS, qui est aussi appelée mezzanine, est une tranche plus risquée qui rapporte des intérêts uniquement lorsque la tranche supérieure a été complètement payée. Elle peut représenter environ $15 \%$ à $30 \%$ de l'ABS et est généralement cotée «BBB ». Ce qui fait qu'elle rapporte généralement un taux d'intérêt de $2 \%$ à $3 \%$ plus élevé. Compte tenu de son risque, elle est beaucoup plus difficile à transférer aux investisseurs qui recherchent davantage la garantie. Finalement, la tranche « équité » est la portion à haut risque qui est payée en dernier et que généralement les émetteurs d'ABS vont plutôt conserver.

Comme le dit Hull, «l'objectif du concepteur de l'ABS est de maximiser le volume de la tranche supérieure sans perdre la note AAA, ce qui optimise le profit réalisé » (Hull, Hénot et Deville, 2014, 185). En créant des montages complexes de prêts, 
ils ont réussi à maximiser cette tranche tout en transférant aux investisseurs une quantité astronomique de prêts "subprimes ». Le tout sans affecter la généreuse cote de crédit AAA, cote garantissant la très haute qualité de ces titres.

Mais ce n'est pas tout. Dans l'optique de vendre le plus de titres possible, les banquiers de Wall Street ont poussé l'audace jusqu'à créer un deuxième titre, l'ABS CDO (Collateralized Debt Obligation). Ils ont pris plusieurs tranches « mezzanines »d'ABS (celles qui sont difficiles à vendre) et les ont regroupés dans un seul et même titre. Le plus étonnant c'est qu'en rassemblant tous ces actifs à risque moyen, on arrivait à créer une tranche supérieure cotée AAA qui pouvait représenter jusqu'à $65 \%$ du portefeuille. En terme simple, on arrivait à créer des actifs de haute qualité en regroupant des actifs de qualité moyenne. Tout ça, sous les yeux des grandes agences de notation de crédit.

\section{Les agences de notation}

Quel est le rôle crucial qu'ont joué ou n'ont pas joué les agences de notation de crédit dans cette crise financière? Normalement, elles auraient dû analyser convenablement les titres créés par les banques et s'inquiéter du risque qu'ils comportaient. Cela aurait permis d'attribuer des cotes de crédit beaucoup moins intéressantes et par le fait même d'avertir convenablement les investisseurs des risques réels de ces produits. Évidemment, cela aurait diminué énormément la demande pour les ABS et les CDO contenant des prêts « subprimes » et aurait forcément endigué la frénésie à laquelle nous avons assisté.

Notons que ces grandes agences étaient auparavant détenues par les professionnels qui y travaillaient. Ils avaient donc à cœur de maintenir la crédibilité de leur organisation pour assurer la continuité de leurs activités. Aujourd'hui, elles sont constituées, pour plusieurs, en sociétés par actions et cotées en Bourse, ce qui les met à tout le moins en apparence de conflit d'intérêts. La pression de rentabilité financière à court terme a certes pu influencer l'analyse de crédit. Et si c'était le cas, accordons-nous alors trop d'importance à ces notations de crédit?

D'une part, nous pourrions aussi estimer que les agences de crédit n'étaient pas bien outillées pour analyser des produits volontairement créés pour être incompréhensibles. Alors qu'elles disposaient des ressources nécessaires pour analyser le crédit des pays et des entreprises, les ressources reliées à l'analyse des titres adossés (ABS et CDO) sont restées relativement faibles et n'ont pas suivi la croissance fulgurante du volume de dossiers. Si celles-ci avaient été suffisantes et compétentes, aurait-on pu déceler les risques cachés derrière ces montages financiers? Aussi, nous pourrions reprocher aux agences de crédit de ne pas avoir mis en place tous les moyens voulus pour mieux comprendre ces produits. Incompétence, laxisme ou les deux?

En somme, un assouplissement du crédit conjugué au transfert du risque vers les investisseurs a permis aux grandes banques d'engranger des profits astronomiques et de mettre la table pour une crise financière sans précédent. Toutefois, l'onde de choc n'aurait pas été aussi grande sans l'utilisation massive de produits dérivés; principalement le swap de crédit CDS (Credit Default Swap) et les CDO synthétiques.

L'arrivée des produits dérivés sur les marchés financiers a non seulement élevé le niveau de sophistication, mais aussi de complexité. La création des produits dérivés était légitime, en ce sens qu'elle venait répondre à un besoin de gestion de risque en offrant une protection au détenteur du bien sous-jacent; que ce soit le producteur de blé, le propriétaire d'une devise, d'un titre de dette ou une action. Ces mêmes produits peuvent aussi être utilisés pour spéculer sur la hausse ou la baisse d'un titre ou d'un marché, ce qui peut créer des situations de pertes importantes comme ce fut le cas en 1994 dans la spectaculaire faillite de la Banque Baring de Londres. Cependant, lorsque le produit dérivé est négocié sur des marchés hors cote où la réglementation est très faible, comme dans le cas des swaps de crédit (CDS), le risque peut alors devenir très grand et contaminer complètement le marché.

Le CDS est un instrument financier dérivé créé au milieu des années 1990 et qui a connu un essor important à partir des années 2000. Selon les statistiques de la Banque des règlements internationaux (BRI), le montant de CDS a pratiquement doublé entre 2006 et 2008 pour atteindre près de 58000 milliards de dollars US au début de l'année 2008. À titre comparatif, le produit intérieur brut des États-Unis pour la même année était de 14713 milliards de dollars US, soit quatre fois moins. 
Le CDS a pour objectif de couvrir le défaut de paiement d'un emprunteur donné. À titre d'exemple, un agent financier (A) prête 10 millions de dollars à l'entreprise (B) au taux d'intérêt de $6 \%$. Évidemment, si l'entreprise (B) fait faillite, l'agent financier (A) pourrait perdre une partie ou la totalité de son capital de 10 millions de dollars. Afin de se protéger contre ce risque, l'agent financier (A) acquiert un CDS de 10 millions de dollars auprès d'un agent financier (C). L'agent financier (A) accepte de donner l'équivalent de $2 \%$ de la valeur du CDS par année à l'agent financier (C). Celui-ci s'engage en retour à rembourser les pertes de l'agent financier (A) en cas de défaut de paiement de l'entreprise (B). Bref, le CDS n'est ni plus ni moins qu'une façon d'assurer des prêts contre le risque de défaut de paiement. En soi, cet outil peut être très utile dans le domaine du crédit afin de réduire l'exposition reliée à d'importants prêts. On peut donc comprendre que pendant la frénésie des "subprimes", plusieurs investisseurs avisés et des institutions financières ont pu avoir recours à ces produits pour réduire le risque relié à des actifs comme les $\mathrm{ABS}$ et les CDO. L'envers de la médaille, par contre, est que l'institution financière qui accepte de garantir le prêt en échange d'une petite prime annuelle, doit débourser un montant important en cas de défaut de paiement.

Même si à la base, les dérivés de crédit ont été créés afin de réduire le risque pour les investisseurs et les banques, les particularités de ce produit en ont fait un élément potentiellement dévastateur pour les marchés financiers. En 2002, dans sa lettre aux actionnaires de Berkshire Hathaway, Warren Buffet n’hésita pas à déclarer que ces produits dérivés pouvaient devenir " des armes financières de destruction massive » (Buffett, 2002, 15). Il aura fallu quelques années seulement pour que la prophétie de Buffet se confirme.

\section{Le CDS : assurance ou spéculation}

Contrairement à l'assurance traditionnelle, l'acheteur d'un CDS n'a pas besoin de détenir le titre financier à assurer pour pouvoir prendre un CDS sur ce titre. C'est comme pouvoir assurer contre le vol ou le vandalisme la voiture de luxe de son patron. Si 10 autres personnes font la même chose, c'est qu'il y a de fortes chances que cela arrive. Alors, si vous croyez que les obligations de l'agent financier (A) seront éventuellement en défaut de paiement, vous pourriez prendre un swap de crédit sur ces obligations. Vous spéculez alors sur la faillite de ce titre en acceptant de payer une prime annuelle qui pourrait vous rapporter, selon notre exemple, jusqu'à 50 fois votre mise si l'agent finan$\operatorname{cier}(\mathrm{A})$ fait faillite rapidement.

Ce genre d'opérations hautement spéculatives, appelées «CDS à nu » est d'ailleurs très bien expliqué dans le livre de Michael Lewis «The Big Short» (Lewis, 2010). Au plus fort de la crise, la spéculation était si élevée qu'il n'était pas rare d'avoir plus de CDS en circulation sur un titre que la valeur réelle de sa dette. Comme le souligne Hull : "Quand Lehman Brothers a fait faillite en septembre 2008, il y avait environ 400 milliards de dollars US de CDS sur le marché, alors que la dette totale avoisinait les 150 milliards de dollars US » (Hull, Hénot et Deville, 2014, 614).

Qui sont ces spéculateurs qui achètent des CDS sur ces dettes « subprimes » qu'ils ne possèdent pas? Les mêmes qui vendent des actions qu'ils ne possèdent pas (short sale) dans l'espoir que le prix baisse. Les mêmes qui vont investir dans une firme sous-évaluée et puis l'obliger à liquider ses actifs pour réaliser un gain à court terme sans faire d'analyse réelle du potentiel de l'entreprise. Les mêmes qui acquièrent une multitude d'actions supplémentaires d'une firme, parce qu'ils en ont la capacité, pour évincer les administrateurs et même la direction afin d'y imposer leur exigence de rendement à court terme, s'ils n'en sont pas satisfaits. Bref, toute cette spéculation a aussi un effet pervers sur les marchés et la gouvernance des entreprises.

Pour que les gens puissent spéculer ainsi, il faut que certaines institutions financières acceptent de jouer le jeu de la contrepartie. Cela a été le cas de la compagnie d'assurance AIG qui est devenue un des joueurs les plus importants à l'échelle mondiale dans les CDS. Elle a accepté de garantir des sommes colossales de titres " subprimes » en échange de primes relativement faibles. Rappelonsnous que malgré un risque important, ces titres disposaient d'une excellente cote de crédit. Ils n'étaient donc pas très coûteux à assurer puisque techniquement le risque de défaut était très faible. Au bout du compte, au plus fort de la crise, AIG a essuyé des pertes de 265 milliards \$ US, beaucoup plus que ce qu'elle pouvait absorber (Truffaut, 2017). Considérée comme un «Too big to fail», en raison de l'impact qu'aurait sa faillite sur l'ensemble du système financier, AIG a été épargnée grâce à 
une intervention rigoureuse du gouvernement américain qui a pris une part importante du capitalactions de la compagnie. Le gouvernement américain a aussi dédommagé les détenteurs de CDS qui avaient des positions gagnantes contre AIG.

Comment se peut-il qu'un intermédiaire financier puisse accepter un tel risque, surtout si la demande de CDS (Credit default swaps) pour les mêmes dettes provient de plusieurs agents différents? Pour l'émetteur du CDS, cela devient une dette hors bilan et un revenu de $2 \%$ figure à ses états financiers. Un revenu sans la contrepartie peut paraître avantageux, mais pas suffisamment pour compenser le risque. Alors pourquoi?

Plusieurs institutions financières ont regroupé les CDS pour former des CDO synthétiques, un titre financier qui génère des flux monétaires correspondant au paiement des primes des CDS. L'acheteur qui achète ce titre reçoit des primes d'assurance et en contrepartie il accepte de garantir le paiement du CDS en cas de défaut de paiement. Un portefeuille de CDS obtient alors une meilleure cote de crédit puisqu'il est peu probable que tous les emprunts garantis par des CDS fassent défaut en même temps. L'institution financière élimine son risque puisqu'elle le transfère à d'autres et l'acquéreur obtient un meilleur rendement en s'engageant à le compenser. Et voilà comment on peut se débarrasser d'un risque qu'on ne veut pas, tout en continuant de réaliser les profits.

Un autre problème que pose le CDS c'est qu'il est négocié sur un marché hors cote, à l'instar des titres à revenu fixe. Ce marché opaque est réservé aux gros investisseurs et l'information n'est pas accessible à tous de façon égale. Par exemple, les institutions financières possèdent de l'information privilégiée sur la situation financière de leurs clients et peuvent être en mesure de participer à des opérations de swap de crédit alors que la contrepartie n'a pas accès à cette information. En terme simple, c'est comme jouer au poker contre un adversaire qui connaît déjà les cartes à venir. En finance, on parle alors d'asymétrie de l'information.

On peut penser que cette asymétrie n'est pas si grave après tout, s'agissant presque uniquement de gros investisseurs institutionnels qui acceptent de jouer le jeu en toute connaissance de cause. Cependant, c'est ce genre d'asymétrie qui a mené des institutions financières à prendre des risques insoupçonnés ou mal évalués et à se retrouver dans des situations de pertes si grandes que les gouvernements ont dû intervenir pour éviter un effondrement complet du système financier. Bref, l'argument de base du capitalisme sauvage qui veut que le plus fort l'emporte et que le plus faible périsse ne peut fonctionner si à chaque fois le gouvernement doit intervenir pour éviter une catastrophe économique.

\section{Crise de confiance}

La dérive des marchés financiers ne repose pas seulement sur la création des CDS, des CDO synthétiques ou des autres artifices mathématiques de la finance. Ces exemples n'expriment qu'un symptôme de cette dérive. Les marchés financiers ne doivent pas être des casinos, mais plutôt un instrument important pour le développement économique et social de collectivités. L'attente de rendement élevé comme jadis ne correspond en rien à la croissance et au contexte économique de cette décade. Les objectifs à courte vue des spéculateurs sont potentiellement très nuisibles et peuvent pousser les sociétés et leurs dirigeants à faire des choix qui ont un effet positif sur le profit à court terme et sur le cours de l'action, mais qui sont néfastes à long terme pour l'organisation. C'est cette vision à court terme, où les institutions financières sont poussées à prendre des risques considérables, qui a mené à la crise financière.

En somme, la crise financière déclenchée en 2007 aura ébranlé solidement les bases de notre système financier tout en ayant aussi porté un dur coup à une des croyances de base de la théorie financière, soit l'efficience des marchés. Cette théorie prétend que les marchés financiers évaluent correctement les titres en fonction de toute l'information disponible et qu'il devient donc impossible de s'enrichir anormalement de façon systématique. C'est cette croyance en l'efficacité suprême des marchés qui est à la base du laisser-aller et de la déréglementation. On constate désormais que les marchés peuvent parfois être complètement irrationnels et dysfonctionnels et un minimum d'interventions et de réglementations est nécessaire pour éviter les dérapages et entraîner ainsi une crise de confiance.

Certes, le gouvernement y est allé d'une intervention musclée pour rétablir la confiance à la suite de la déroute de 2008 en injectant des milliards de dollars dans certaines institutions financières qui 
avaient subi des pertes énormes. La valeur des produits financiers sophistiqués avait été fortement dévaluée sur les marchés et, en fonction des normes comptables existantes pour établir la valeur des actifs au marché, les institutions financières ont enregistré des pertes importantes, mais souvent non réalisées. Conséquemment, dès l'année suivante, plusieurs institutions financières ont enregistré des profits records. Ceci démontre bien que les interventions a posteriori sont nécessaires pour éviter l'effondrement du système financier; mais en réalité, cela peut aussi laisser l'impression que comme au casino, la banque gagne toujours.

\section{La place de l'industrie financière dans l'économie}

Il y a d'ailleurs lieu de se poser la question sur la place qu'occupe l'industrie financière par rapport au reste de l'économie (figure 1). La part des profits des entreprises manufacturières par rapport à l'ensemble de l'économie aux États-Unis est en déclin et celle des entreprises financières est en croissance.
Selon Allaire et Firsirotu : «Mutatis mutandis, the same proportional phenomenon plays out in all developed country (with the relative exception of Germany)» (Allaire et Firsirotu, 2009, 62).

La décroissance de la part du secteur manufacturier américain passant de $52 \%$ en 1937 à $22 \%$ en 2016 pourrait s'expliquer en partie par la délocalisation des unités de production, mais il est incontestable que la part des profits du secteur financier a doublé pour cette même période. Il y a donc lieu de se questionner sur l'importance de la valeur sociétale qu'engendre l'industrie financière par rapport à celle du secteur manufacturier. Nul doute que le secteur financier est nécessaire pour notre économie, mais sa prise de profit grandissante serait plus créatrice de valeur collective si elle était créée dans le secteur manufacturier qui est plus bénéfique pour les collectivités, car plus créatrice d'emploi. Surtout lorsque cette prise de profits se fait sur le dos des ménages à faible revenu, comme ce fut le cas en 2008.

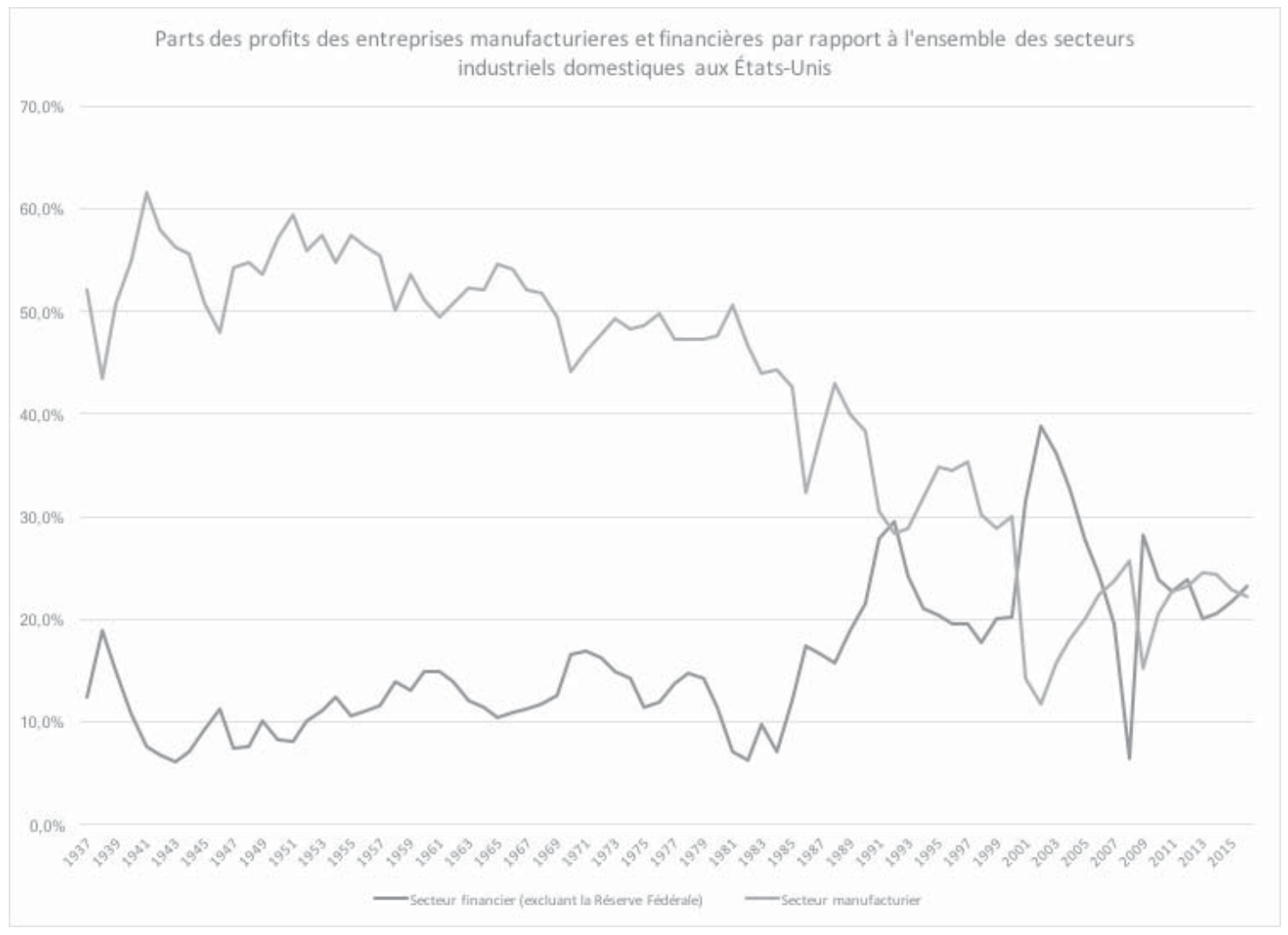

Figure 1 - Parts des profits des entreprises manufacturières et financières par rapport à l'ensemble des secteurs industriels domestiques aux États-Unis

Source : U.S. Bureau of Economic Analysis, "Table 6.16 Corporate Profits by Industry" 
Il y a lieu de se questionner sur le rôle réel que jouent les marchés financiers dans notre économie et surtout le rôle que nous voudrions qu'ils jouent. Si on veut un marché plus juste, plus équitable, il faut modifier les règles pour mieux contrôler le risque que prennent les institutions financières et s'assurer de protéger les petits investisseurs, les entreprises et les gouvernements de la spéculation des gros joueurs de ce monde.

Certaines actions ont d'ailleurs été entreprises un peu partout dans le monde pour resserrer les règles du crédit immobilier et assurer une meilleure solvabilité des banques. On a aussi entrepris de légiférer afin de mieux baliser l'utilisation de produits dérivés comme les CDS. À titre d'exemple, l'Union européenne a passé une loi en 2012 visant à interdire les opérations de CDS à nu contre les obligations d'États européens. Ainsi il devient plus difficile pour un investisseur de spéculer sur le défaut de paiement d'un pays européen.

Cependant, les efforts pour réglementer davantage les opérations financières se heurtent à une forte résistance. D'abord, c'est une question de gros sous. Les grandes places financières du monde se font concurrence pour attirer les institutions financières et les capitaux qui en découlent. Dans ce contexte, une réglementation trop sévère pourrait avoir un effet dissuasif et entraîner une fuite de capitaux. En ce sens, il faut que les efforts de réglementation et de contrôle soient concertés à l'échelle internationale afin d'être plus efficaces et de mieux baliser un système financier qui s'est mondialisé.

D'un autre côté, on pourrait croire que les tentatives de légiférer les marchés financiers vont nuire à la création de richesse et à la croissance économique. C'est du moins l'opinion des défenseurs du libéralisme économique et de la déréglementation. Si l'on regarde la croissance spectaculaire des marchés boursiers, principalement celui du secteur financier au cours des années 1990 et 2000, cela donne effectivement des arguments de poids à cette thèse. Cependant, il serait intéressant de pousser l'analyse plus loin afin de vérifier si les mesures visant à contrôler les marchés ont un impact réel sur la croissance économique et la création d'emploi.

Il est dangereux de confondre création de richesse et croissance économique. La déréglementation a permis aux banques de croitre à un rythme impressionnant et de grossir à un point tel qu'elles deviennent « Too big to fail ». Les profits engendrés par ces monstres financiers auront permis aux dirigeants et à certains conseillers en placement de toucher des salaires et des bonis souvent indécents. L'utilisation massive de CDS leur aura même permis de spéculer de façon honteuse sur la faillite des produits qu'ils ont eux-mêmes créés. Mais tous ces milliards qui ont disparu des coffres des banques ont eu un effet catastrophique sur l'économie mondiale qui peine encore à retrouver la vigueur d'avant 2008 .

Bref, l'histoire récente démontre le danger d'une confiance aveugle dans les marchés financiers. Il faut créer des instances de contrôle et de réglementation afin de s'assurer de baliser davantage les opérations financières et principalement celles portant sur les produits dérivés qui se négocient hors cote, dans le but d'éviter des dérapages comme celui de 2008. On peut s'interroger à la fois sur l'intérêt d'imposer une réduction de l'utilisation de ces produits, ou encore créer une chambre de compensation ou un marché particulier, lorsqu'ils atteignent un certain volume et sur les dangers que représente la très forte concentration de capitaux dans les mains de quelques institutions financières qui peuvent prendre des risques considérables, sans toutefois avoir à s'inquiéter des conséquences. Parier sur la baisse de la valeur d'une entreprise, d'une dette nationale ou d'un titre provoque inévitablement cette situation. À la lumière de ce qui précède, y a-t-il d'autres choix que d'interdire ou du moins de limiter ce genre d'actions, au risque de voir l'histoire se répéter...

\section{RÉFÉRENCES}

Allaire, Y. et Firsirotu, M. E. (2009). Black markets-- and business blues: the man-made crisis of 2007-2009 and the road to a new capitalism (1 éd.). Montréal, Québec : FI Press, 62.

Autorité des marchés financiers (2013). Les obligations et dispenses d'inscription et les obligations continues des personnes inscrites, 31-103.

Buffett, W. (2002). Berkshire Hathaway Shareholders Letters. 
Chase National Bank of the City of New York (1933). United States. Banking act of 1933 (Glass-Steagall banking bill) approved by the President June 16th, 1933. New York, NY : The Chase National Bank of the City of New York.

Christopher, J. (1993). À Londres : la réforme de l'organisation financière. Revue d'économie financière, 25(2), 227-31.

Hull, J., Roger, P., Hénot, C. et Deville, L. (2014). Options, futures et autres actifs dérivés. (9e éd.). Montreuil, France : Pearson France.

Lewis, M. (2010). Le casse du siècle. Paris, France : Sonatine.

Truffaut, S. (2017). Anatomie d'un désastre : la crise financière de 2008, de Reagan à Trump. Montréal, Québec : Éditions Somme toute. 